\title{
SISTEMA PARA MEDIÇÃO ON-LINE DE CAMADA DE ÓLEO NA SUPERFÍCIE DE TIRAS - EMG SOLID*
}

Anderson Rosa Pereira ${ }^{1}$

\section{Resumo}

O trabalho visa apresentar dois tipos de equipamentos para medição on-line de camada de óleo em superfícies de chapas e placas. O equipamento é denominado SOLID® (Surface Oil Layer Inline Determination) e tem como aplicação típica laminadores, processadores de tiras e qualquer outro tipo de linha onde algum tipo de óleo ou cera é aplicado na superfície do material. O equipamento realiza medição on-line com o material em produção e determina a camada de óleo ou cera em toda a largura e comprimento do material, permitindo ao cliente monitorar com precisão a aplicação deste produto.

Palavras-chave: Medição de camada de óleo ou cera; Determinação on-line de camada de óleo ou cera; Visualização gráfica da camada aplicada de óleo ou cera.

\section{SYSTEM FOR ONLINE MEASUREMENT OF OIL LAYER ON THE SURFACE OF STRIPS - EMG SOLID}

\section{Abstract}

This work presents two types of equipment for online measurement of oil layer on surfaces of strips and plates. The equipment is denominated SOLID® (Surface Oil Layer Inline Determination) and it has as a typical application rolling mill, strip processors and any other kind of line where some type of oil or wax is applied at the surface of the material. The equipment performs online measurement with the strip running and determines the oil or wax layer over the entire width and length of the material, allowing the customer to precisely monitor the application of these products. Keywords: Measurement of oil or wax layer; Online determination of oil or wax layer; Graphical visualization of oil or wax layer applied.

1 Engenheiro Eletricista, Coordenador de Vendas, Departamento de engenharia, EMHEletromecânica e Hidráulica Ltda, Belo Horizonte, Minas Gerais, Brasil. 


\section{INTRODUÇÃO}

A crescente demanda por novos materiais e componentes cada vez mais complexos requerem processos cada vez mais confiáveis. A camada de óleo também se tornou extremamente importante para conformação de produtos laminados de alumínio e aço. As exigências cada vez maiores em relação a uma camada de lubrificante uniforme e definida são particularmente evidentes na indústria automobilística.

O EMG SOLID® (SOLID® = Surface Oil Layer Inline Determination) é um sistema usado para medição on-line de camadas de óleo com a tira em movimento e é feita por toda a largura e comprimento do material.

A EMG Automation $\mathrm{GmbH}$ oferece dois tipos de tecnologia para medição do peso superficial da camada de lubrificante. O sistema EMG SOLID® IR usa o recurso de espectroscopia por infravermelho enquanto o sistema EMG SOLID® LIF usa a espectroscopia de fluorescência induzida por laser. Ambas as tecnologias tem suas vantagens e desvantagens dependendo da aplicação para o qual são utilizadas, pelo que o sistema correto é oferecido de acordo com a respectiva aplicação.

\section{DESCRIÇÃO DO SISTEMA}

\subsection{Descrição do sistema EMG SOLID® IR}

\subsubsection{Princípio de funcionamento}

O princípio de medição real implementado pelo EMG SOLID® é baseado na lei Lambert-Beer, que é descrita na seguinte fórmula.

$$
I=I o e^{-\varepsilon d}
$$

- I: Intensidade da luz transmitida, unidade:

$$
\frac{W}{\mathrm{~cm}^{2}}
$$

- Io: Intensidade da luz incidente (irradiada), unidade:

$$
\frac{W}{c m^{2}}
$$

- $\boldsymbol{\varepsilon}$ : Coeficiente de absorção, unidade:

$$
m^{-1}
$$

- d: Espessura da camada do meio penetrado, unidade:

$\mathrm{m}$

Todas as moléculas exibem absorções características no espectro infravermelho, onde podem ser claramente identificadas e distinguidas do seu ambiente. O princípio básico é a iluminação de uma amostra, como ilustrado na figura 1, com radiação térmica e subsequente análise da luz transmitida. A espessura da camada (d) é descrita pela relação logarítmica da radiação incidente (I0) com a radiação emergente (I) depois de passar através de um meio. 


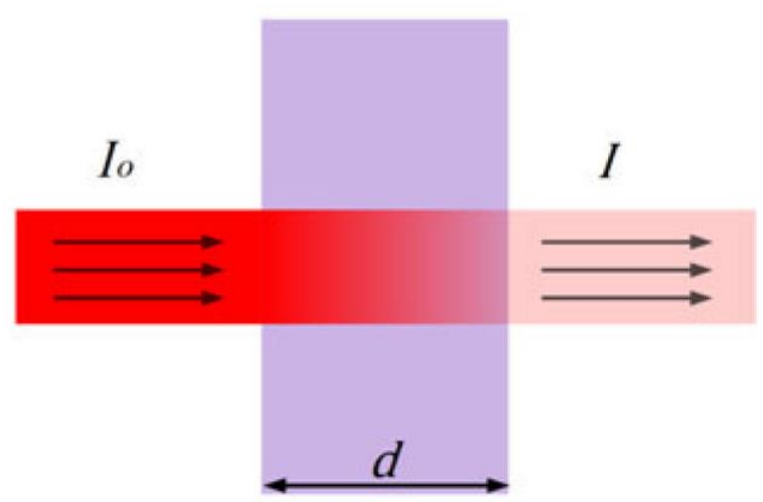

Figura 1. Absorção de sinal

O sistema EMG SOLID® IR utiliza lâmpadas alógenas para emitir radiação infravermelha na superfície do material. Durante este processo, os feixes de luz primeiro penetram a camada de óleo, pelo que uma proporção de sua intensidade é absorvida pelo óleo, antes de ser espalhada difusamente na superfície metálica áspera (não refletida exclusivamente de maneira direcionada), como pode ser visto na figura 2. A caminho do sensor, os feixes de luz penetram a camada de óleo uma segunda vez. As intensidades de comprimentos de onda específicos do espectro de luz são atenuadas pelo óleo novamente nesta configuração.

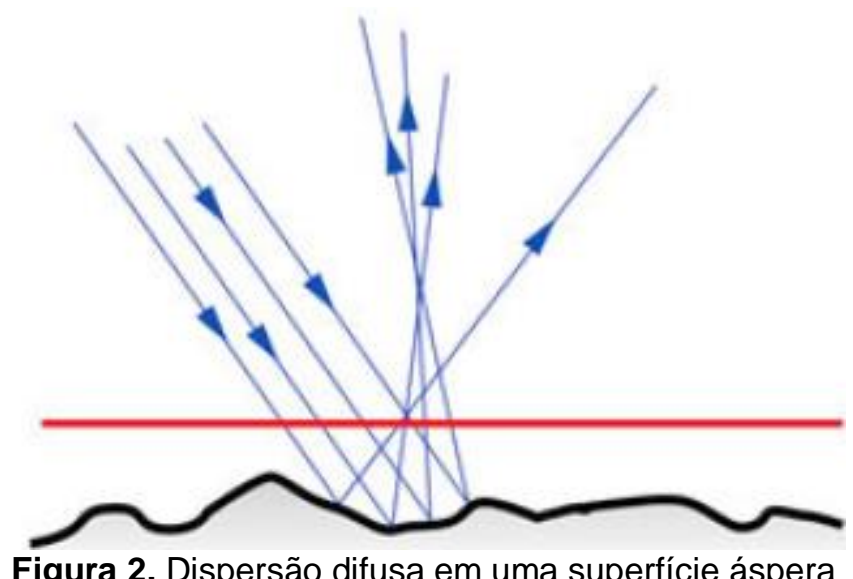

As absorções específicas de substâncias não só mostram a diferenciação da substância a ser medida a partir da superfície, mas também permitem tirar conclusões quanto ao volume da substância com base em sua intensidade. Por conseguinte, pode ser implementada uma medição livre de contato da espessura da camada.

\subsubsection{Arquitetura do sistema e componentes}

A cabeça de medição é o principal componente do sistema e entrega os valores brutos para o controlador. A camada de óleo é calculada a partir dos valores brutos por meio de software especial dentro do painel de controle do EMG iCON® XE com base nos dados de calibração salvos.

As cabeças de medição são montadas em uma unidade de deslocamento eletromotriz e podem ser movidas independentemente uma da outra. A operação manual é ativada através de um painel de controle separado, que está montado ou 
configurado nas proximidades. Quando o modo manual é selecionado, a cabeça de medição pode ser conduzida para uma posição especificada através de um seletor. O sistema de medição EMG SOLID® também pode ser operado através do PC Industrial server, que é montado no painel de controle. O processo de medição pode ser iniciado através de certos sinais do sistema (por exemplo, mudança de bobina, velocidade mínima da tira, tensão da tira, produção de produtos, etc.), o que significa que o sistema de medição pode ser operado de forma totalmente automática sem a intervenção dos operadores de sistema. A comunicação entre a unidade de deslocamento e o armário de controle é realizada exclusivamente por meio de sistemas de rede (Ethernet ou Profibus DP), o que significa que o painel de controle pode ser configurado praticamente em qualquer lugar.

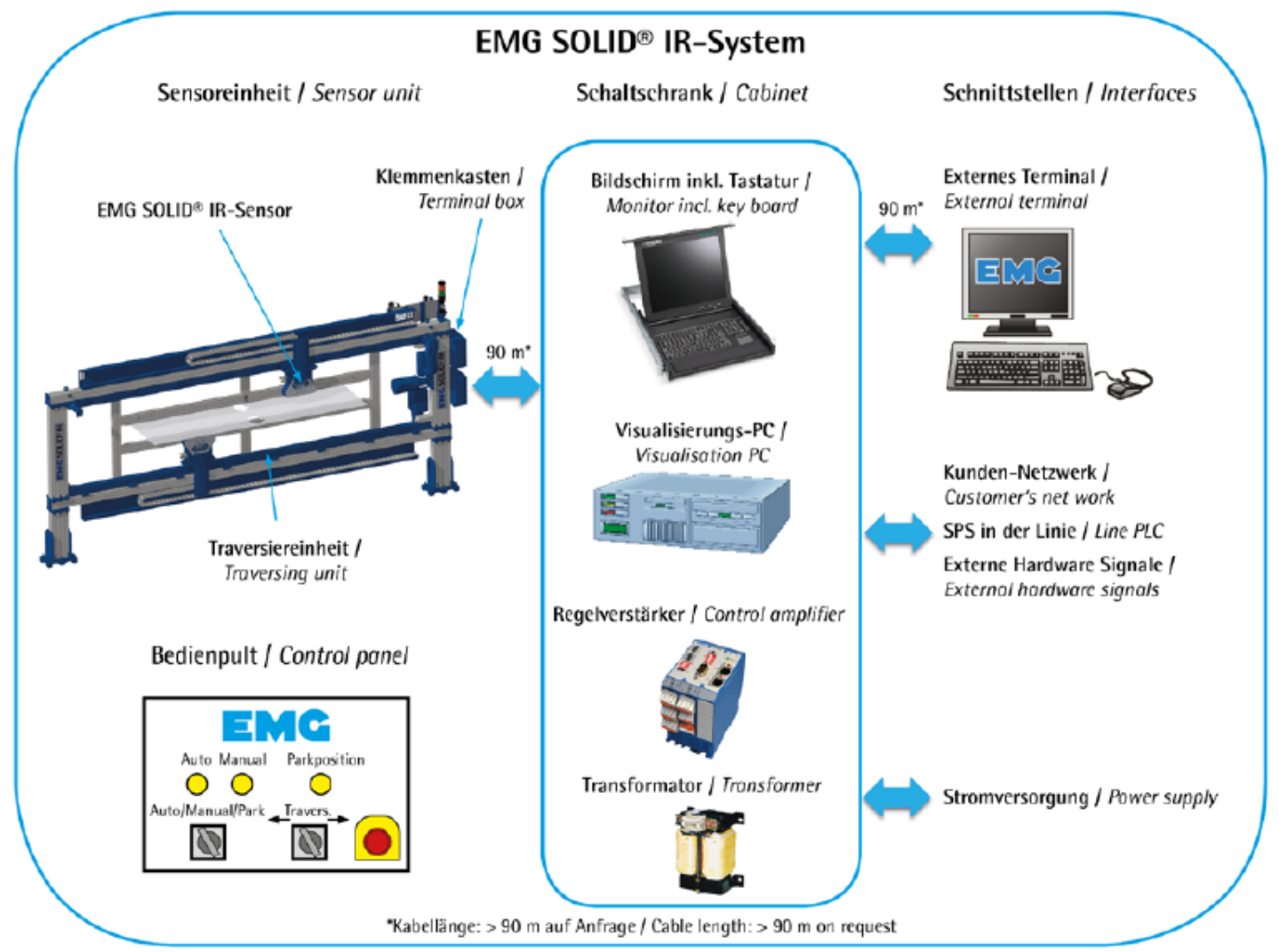

Figura 3. Estrutura do sistema

\subsection{Descrição do sistema EMG SOLID® LIF}

\subsubsection{Espectroscopia de fluorescência induzida por laser}

A espectroscopia de fluorescência induzida por laser (LIF (t)) é convertida em um arranjo especial no sistema EMG SOLID® LIF, como mostrado na figura 4. As moléculas do meio a ser detectado são excitadas com um laser UV através de um guia de ondas ótico e a fluorescência é direcionada para o fotomultiplicador através de uma segunda fibra, onde é detectada. 


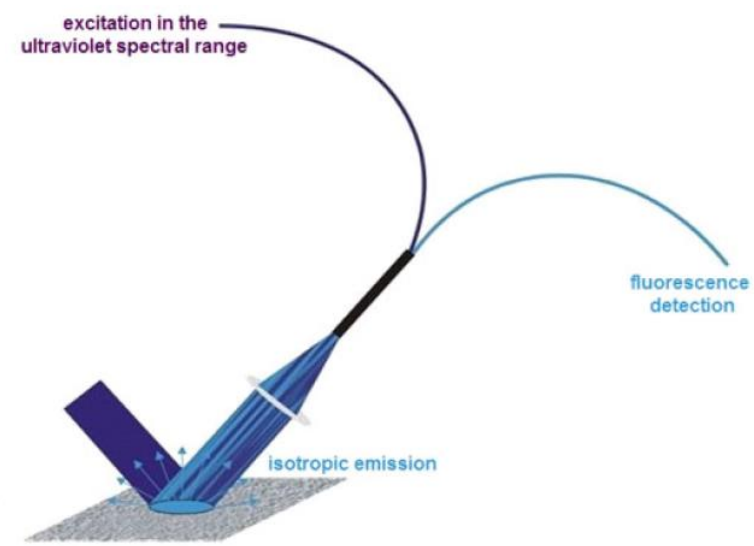

Figura 4. Representação esquemática da configuração de medição

Um laser especial de estado sólido entrega 10.000 pulsos individuais por segundo, estimulando assim a fluorescência do meio a ser medido. A fluorescência é um processo de emissão de luz que, como mostrado na Figura 5, desencadeia a absorção de radiação de excitação de comprimentos de onda adequados e é uma das interações mais eficientes entre luz e matéria. A fluorescência emitida por uma molécula ou átomo imediatamente após a excitação óptica é específica da substância em sua distribuição de intensidade espectral e ainda é altamente detectável, mesmo em quantidades muito pequenas. Por conseguinte, uma medição de fluorescência é particularmente adequada para analisar as camadas mais finas e as menores concentrações. Ao mesmo tempo, deve-se ter em mente que as propriedades de fluorescência de diferentes substâncias e meios de substrato podem diferir umas das outras, o que significa que as calibrações específicas da aplicação são necessárias para fins específicos.

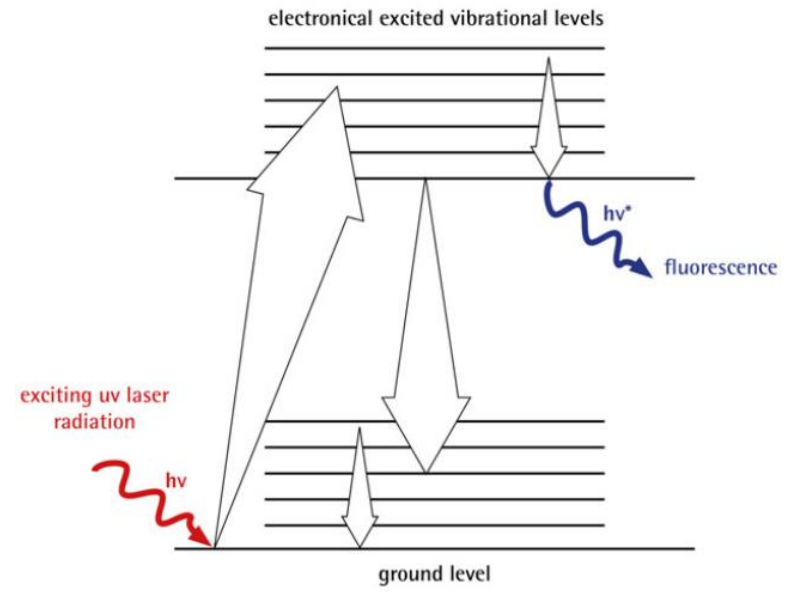

Figura 5. Interação entre luz e matéria: Absorção e fluorescência

\subsubsection{Princípio de funcionamento}

A sequência cronológica que ocorre após um pulso laser é mostrada na figura 6 . 0 período de tempo entre o pulso de luz no laser de excitação e o primeiro foton de fluorescência no sistema detector é o atraso do gatilho ou o atraso básico. O período de tempo entre o pulso de luz no laser de excitação e o primeiro fóton de fluorescência no sistema detector é o atraso do gatilho ou o atraso básico.

Quando o pulso laser desaparece (tipicamente $<0,5$ a $0,7 \mathrm{~ns}$ ), os eventos de fluorescência que ocorrem são contados dentro dos intervalos de tempo I1 e I2. 
Quando a fluorescência desaparece o sinal de luz ambiente é gravado no terceiro intervalo de tempo 13 e está disponível para posterior compensação do sinal útil em 11 e 12.

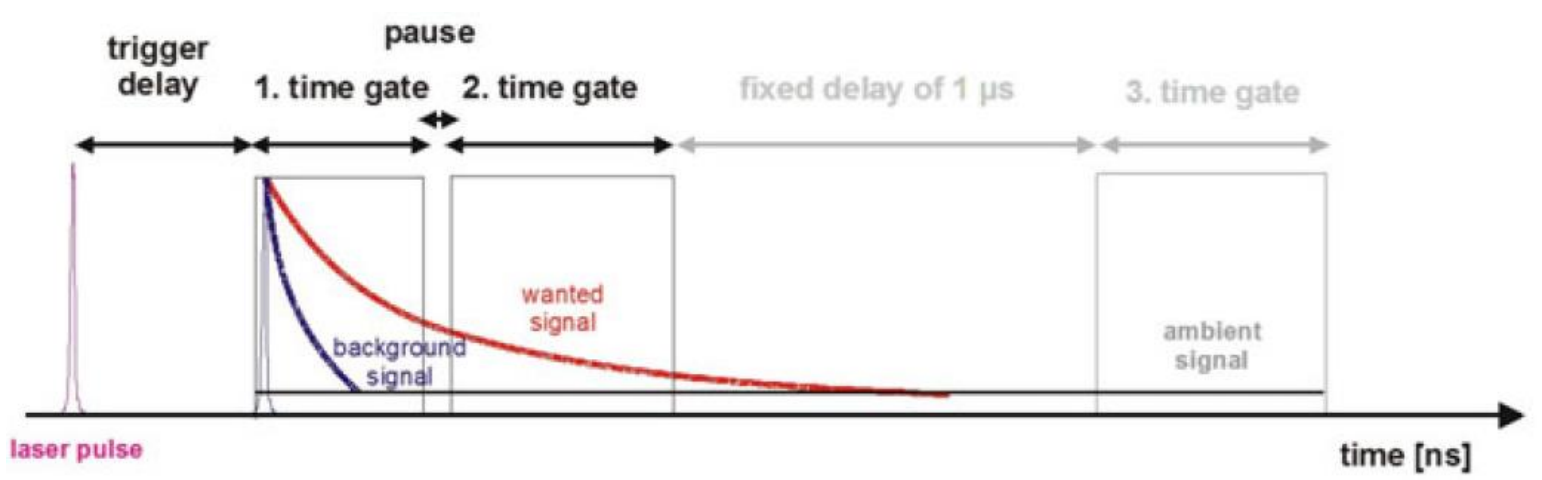

Figura 6. Sequência cronológica de aquisição de sinal após um único pulso laser

\subsubsection{Arquitetura do sistema e componentes}

A implementação técnica da espectroscopia de fluorescência resolvida no tempo induzida por laser [LIF (t)] consiste nos componentes mostrados na figura 7. O laser de microchip UV tipicamente emite pulsos de laser a $10 \mathrm{kHz}$, que são guiados para a cabeça de medição através de uma guia de onda óptica, onde atacam a superfície a ser examinada. A substância a detectar, que está localizada na superfície, absorve a energia do pulso laser e, por sua vez, emite radiação óptica com energia reduzida. Esta radiação, chamada de fluorescência, é emitida uniformemente em todas as direções espaciais e é guiada a partir da guia de onda óptica de detecção para o sistema detector (fotomultiplicador).

O período de referência do pulso laser excitante e a fluorescência detectada no sistema detector são produzidos através da unidade de gatilho e o sinal de fluorescência correspondente é contado através do sistema contador nos respectivos intervalos de tempo.

O sistema detector consiste em um pacote de filtros ópticos, que são adaptados à tarefa de medição, e um fotomultiplicador para detectar eventos de fotons individuais, isto é, menores quantidades de luz. O sinal de fluorescência agora é avaliado através da contagem estatística de fótons de fluorescência nos intervalos de tempo após cada pulso laser.

A camada de óleo é calculada a partir dos valores brutos por meio de software especial no microcontrolador EMG SOLID® iCON dentro do painel de controle com base nos dados de calibração salvos.

As cabeças de medição geralmente são montadas em uma unidade de deslocamento eletromotriz e podem ser movidas independentemente uma da outra. A operação manual é ativada através de um painel de controle separado, que é montado ou configurado próximo da estrutura de medição. Quando o modo manual é selecionado, o cabeçote de medição pode ser conduzido para uma posição especificada.

O sistema de medição EMF SOLID® LIF também pode ser operado através de PC industrial, que poderá ser montado no painel de controle. O processo de medição pode ser iniciado através de certos sinais do sistema (por exemplo, mudança de bobina, velocidade de tira mínima, tensão de tira, produção de produtos, etc.), o que significa que o sistema de medição pode ser operado de forma totalmente automática sem a intervenção dos operadores. Uma interface gráfica do usuário é 
usada aqui no sistema operacional Windows Server. A comunicação entre a unidade de deslocamento e o painel de controle é realizada exclusivamente por meio de redes (Ethernet ou Profibus DP), o que significa que o painel de controle pode ser configurado praticamente em qualquer lugar.

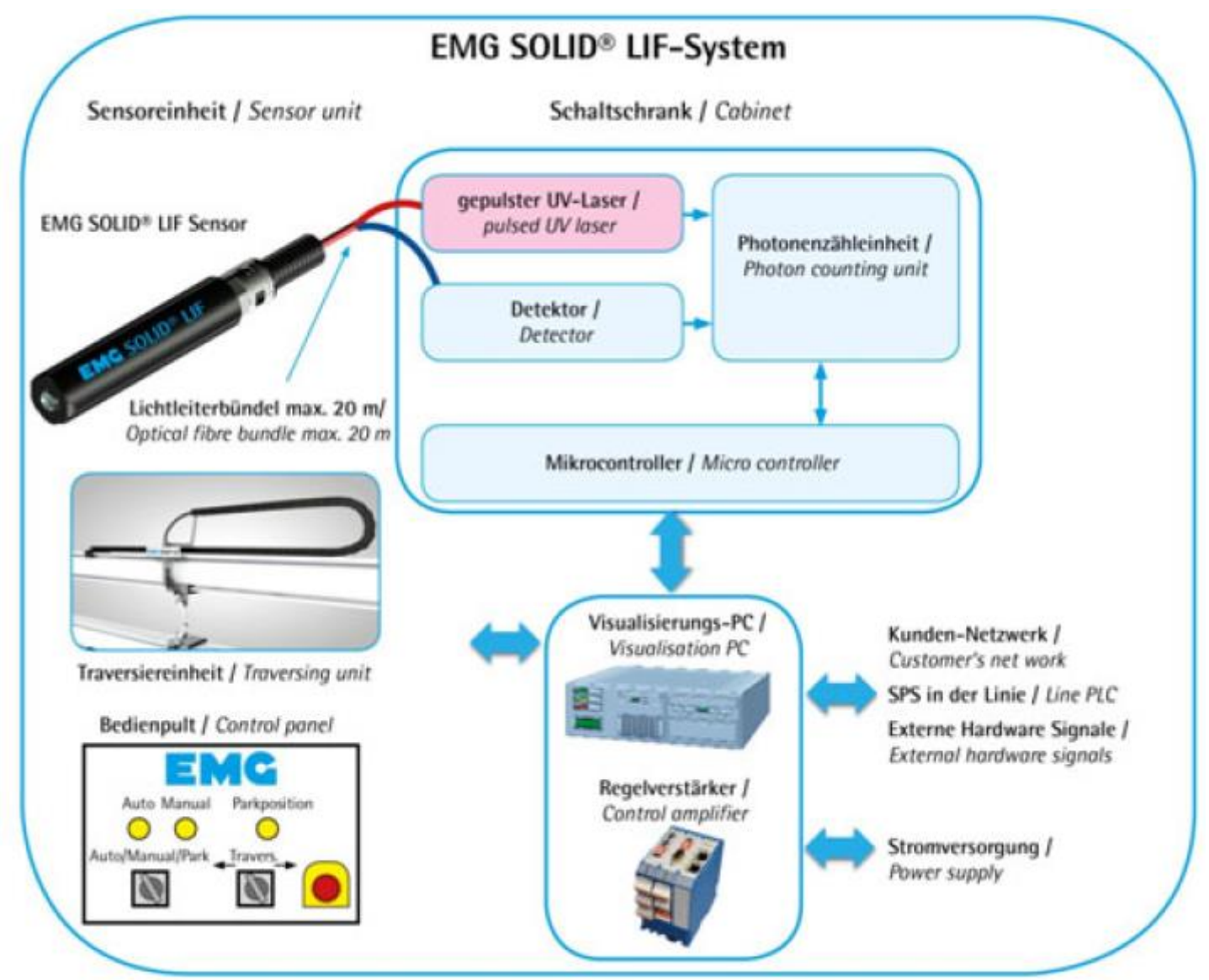

Figura 7. Estrutura do sistema EMG SOLID® LIF

\section{VISUALIZAÇÃO DOS RESULSTADOS MEDIDOS}

Independentemente da tecnologia escolhida (SOLID® LIF ou IR) o pacote de visualização é o mesmo.

O software de visualização EMG SOLID® DATA funciona como Graphical User Interface (GUI) e está instalado em um servidor industrial, que está alocado no painel de controle.

A GUI exibe os valores da camada de óleo em um monitor com resolução Full HD. A sequência de medição, o programa de deslocamento e os ciclos de monitoramento do equipamento de teste são configurados de acordo com as especificações do cliente através do software EMG SOLID®. 

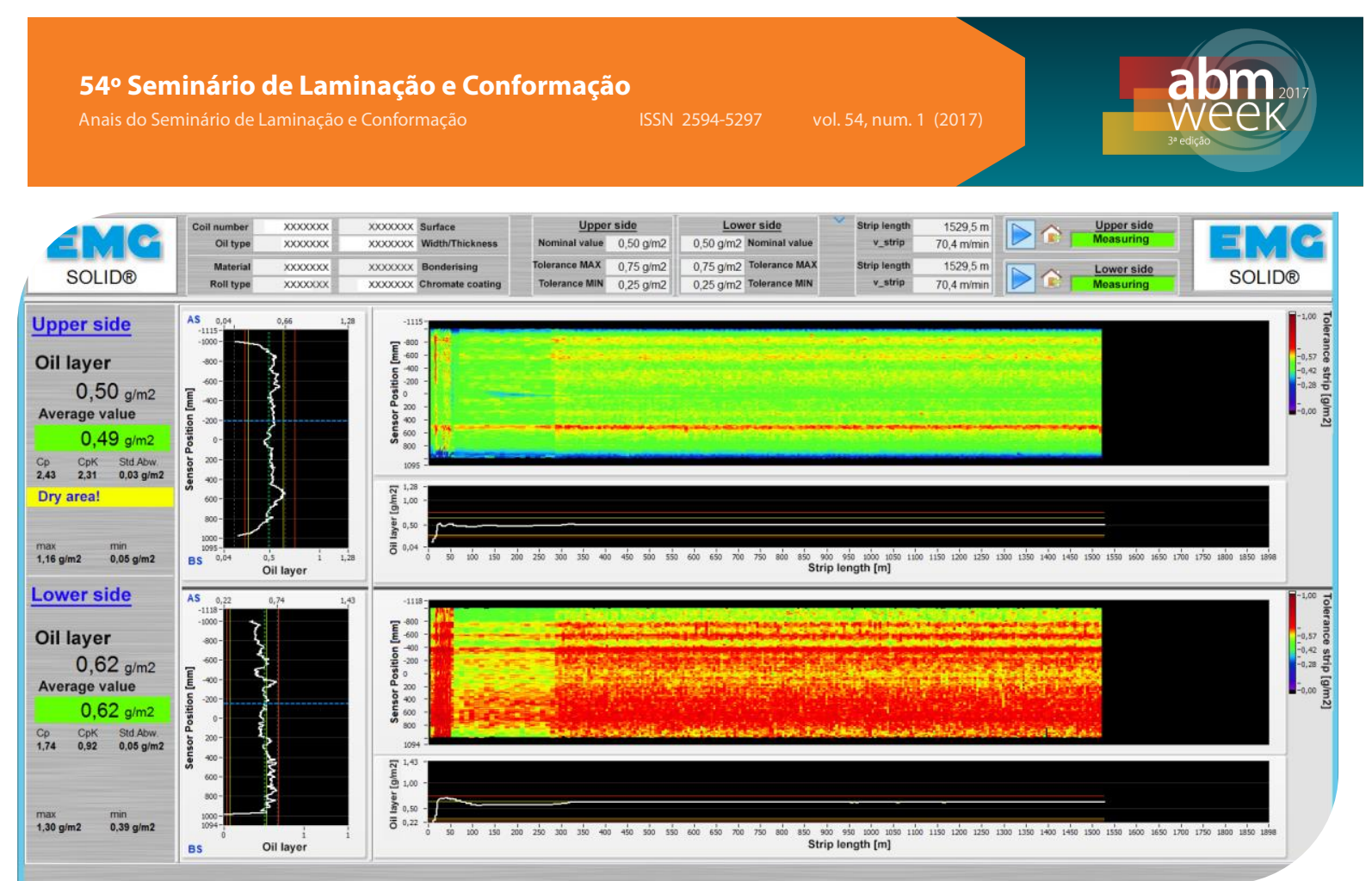

Figura 8. Visualização do valor medido da camada de óleo (medição em ambos os lados)

A Figura 8 mostra como os dados de camada de óleo são exibidos graficamente e numericamente em $\mathrm{g} / \mathrm{m}^{2}$ dentro da interface do usuário. Todos os dados medidos relevantes podem ser transferidos em paralelo para a rede do cliente. Os valores de referência e valores limite são especificados, exibidos e monitorados visualmente pelo cliente. Se os valores limite não forem atingidos ou forem excedidos, um aviso visual será exibido no sistema. Também é possível emitir um sinal sonoro ou ativar um alerta luminoso para aviso.

\section{AREAS DE APLICAÇÃO}

O espectro de aplicação típico do sistema de medição SOLID® da EMG varia desde os laminadores, onde a aplicação inicial de óleo é implementada, em linhas de processamento e beneficiamento do material, em linhas onde a lubrificação é necessária para o processo de conformação e por fim em linhas onde é necessário verificar a ausência de óleo antes de processos de revestimento.

Áreas típicas de aplicação:

- Laminadores

- Linhas de galvanização a quente

- Linhas de processamento e beneficiamento de tira

- Linhas de corte longitudinal

- Linhas de corte transversal

- Linhas de inspeção

- Prensas

- Linhas de Blanks 


\section{CONCLUSÃo}

Com a utilização deste tipo de equipamento o usuário ganha uma poderosa ferramenta para visualizar a camada de óleo por toda a superfície do material produzido. Com isto é possível apurar ganhos no tocante a melhora na estabilidade e confiabilidade do processo, minimização de sucata, verificação constante de possíveis falhas na oleadeira, controle perfeito do material de saída, evitar reclamações de clientes por fornecimento de material fora das especificações, possibilidade de fornecer os registros de qualidade relativos à camada de óleo ao cliente final e tantos outros.

\section{REFERÊNCIAS}

1 Verband der Automobilindustrie e.V. (VDA)(German Association of the Automotive Industry). VDA Test Specification 230-201: Prelubes. 2003.

2 Losch, A.: Prelubes for sheet metal forming. In Tribology 2000 Plus, $12^{\text {th }}$ International Colloquium Tribology, Fuchs Europe Schmierstoffe GmbH, January 11-13, 2000, Ostfildern.

3 Gemmer T. SOLID IR Specification. 2017. Volume 1: 5 a 9.

4 Gemmer T. SOLID LIF Specification. 2017. Volume 1:5 a 12. 